\title{
TEMPO DE VIDA ÚTIL DE PROPELENTES BASE-SIMPLES
}

Jony Andrade*, Koshun Iha e Jose Atílio Fritz Fidel Rocco

Instituto Tecnológico de Aeronáutica, Praça Marechal Eduardo Gomes, 50, 12228-900 São José dos Campos - SP, Brasil

Glaci Ferreira Martins Pinheiro e Enézio Donizetti Moreira

Instituto de Aeronáutica e Espaço, Praça Marechal Eduardo Gomes, 50, 12228-900 São José dos Campos - SP, Brasil

Maria Encarnación Vázquez Suárez-Iha

Instituto de Química, Universidade de São Paulo, Av. Prof. Lineu Prestes, 748, 05508-900 São Paulo - 'SP/Centro de Ciências e Humanidades, Universidade Presbiteriana Mackenzie, Rua da Consolação, 930 01302-970 São Paulo - SP, Brasil

Recebido em 15/3/07; aceito em 3/9/07; publicado na web em 10/3/08

\begin{abstract}
SAFE SHELF LIFE OF SINGLE-BASE PROPELLANTS. The purpose of this work was to determine the safe shelf life of single-base propellants. The kinetic parameters relative to the consumption of the stabilizer diphenylamine (DPA) added to the propellant were determined as a function of the storage and ageing time. High Performance Liquid Chromatography (HPLC) with spectrophotometric detection was used to determine the DPA percentage before and after the artificial ageing at 60,70 and $80{ }^{\circ} \mathrm{C}$. The experimental data were very well adjusted to a pseudo-first order kinetic model and the respective kinetic constants are $8.0 \cdot 10^{-3}$ day $^{-1}\left(60{ }^{\circ} \mathrm{C}\right) ; 1.9 \cdot 10^{-2}$ day $^{-1}\left(70^{\circ} \mathrm{C}\right) ; 1.2 \cdot 10^{-1}$ day $^{-1}\left(80^{\circ} \mathrm{C}\right)$. The activation energy was calculated as $130-\mathrm{kJ} \mathrm{mol}^{-1}$ and the half-time for depletion of the DPA at the hypothetical temperature of $40^{\circ} \mathrm{C}$ of storage was estimated as being 6 years.
\end{abstract}

Keywords: kinetics; diphenylamine; single-base.

\section{INTRODUÇÃO}

A nitrocelulose, Figura 1, é um composto muito empregado na obtenção de propelentes sólidos, em decorrência do seu alto poder energético ${ }^{1}$ e da baixa emissão de fumaça quando em combustão, originando o propelente sólido conhecido como base simples (BS). ${ }^{2}$ Entretanto, em virtude da sua sensibilidade a variações de temperatura, processos de decomposição química podem ocorrer ao longo do período de armazenamento, transporte e manuseio diminuindo, assim, o tempo de vida útil do grão propelente. ${ }^{3}$ Este processo de degradação ${ }^{4}$ deve-se principalmente à ruptura das ligações $\mathrm{O}-\mathrm{NO}_{2}$ com a formação de radicais $\mathrm{NO}_{2}^{*}$ e $\mathrm{NO}^{\circ}$. Além disso, tem-se também a reação entre os produtos de decomposição, com a própria nitrocelulose, produzindo o fenômeno conhecido como decomposição autocatalítica. $^{5}$

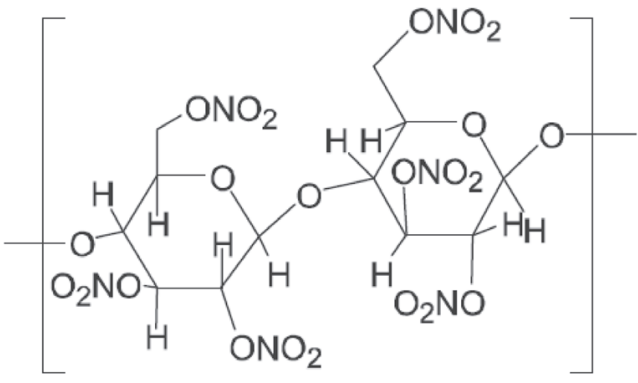

Figura 1. Estrutura química da nitrocelulose ${ }^{l}$

A decomposição autocatalítica é responsável por ocorrências como diminuição do potencial energético do grão propelente, instabilidades de queima durante o processo de combustão e, em casos extremos, auto-ignição durante o período de armazenamento. ${ }^{6}$ Em decorrência disto, é necessário que espécies químicas estabili-

*e-mail: jonyquim@ita.br zantes sejam adicionadas à formulação, de forma a inibir este processo de degradação. ${ }^{7}$

A difenilamina (DPA) está entre os estabilizantes mais empregados para formulações de propelentes à base de nitrocelulose. ${ }^{8} \mathrm{O}$ mecanismo de ação da DPA decorre do fato de o átomo de hidrogênio, ligado ao nitrogênio, reagir facilmente com os grupos $\mathrm{NO}_{\mathrm{x}}$ liberados pela nitrocelulose, ${ }^{9}$ inibindo a aceleração do processo de decomposição do BS. ${ }^{10}$ Deste modo, o conhecimento da cinética de consumo do estabilizante ${ }^{11}$ para propelentes à base de nitrocelulose oferece uma boa base de dados para a projeção do tempo durante o qual o BS pode ser armazenado com segurança ("safe shelf life ou storage life").

$\mathrm{Na}$ literatura são encontrados alguns trabalhos sobre a cinética de consumo de estabilizantes do tipo DPA em formulações de propelentes base-simples. Entretanto, em virtude destes materiais serem empregados em áreas restritas, normalmente de emprego militar, detalhes em relação à origem da amostra, se obtida em laboratório ou na indústria; dados quanto à forma física; teor de nitração e a presença de aditivos na formulação, normalmente são omitidos. Estes parâmetros são importantes no estudo cinético de decomposição de formulações de propelentes, pois atuam diretamente no processo. ${ }^{12}$ Desta forma, a princípio os parâmetros obtidos na literatura podem ser utilizados como referências, mas, neste caso, em se tratando de amostras reais de grãos propelentes, uma análise em particular é recomendável.

Este trabalho teve como objetivo a determinação dos parâmetros cinéticos que representam o consumo de estabilizante para uma amostra de propelente BS produzida em escala industrial, por meio de envelhecimento artificial e utilizando a cromatografia líquida de alto desempenho (HPLC) para uma projeção do tempo de vida útil do grão.

\section{PARTE EXPERIMENTAL}

\section{Material utilizado}

Para a realização deste estudo foi utilizada uma amostra de 
propelente base-simples fornecida pela empresa IMBEL do Brasil, registrado como BS REX 1200. Para o envelhecimento artificial isotérmico do propelente foram utilizados tubos de vidro pirex e blocos de aquecimento com controle automático de temperatura. Foram utilizados ainda diclorometano p.a. de marca Vetec, acetonitrila grau HPLC da J.T. Baker, difenilamina p.a (DPA) e materiais comuns de laboratório.

\section{Envelhecimento térmico}

O envelhecimento do grão propelente $\mathrm{BS}$ no modo isotérmico foi realizado fixando-se as temperaturas de 60,70 e $80^{\circ} \mathrm{C}$ por um período de tempo programado. Alíquotas de $20 \mathrm{~g}$ de amostra foram acondicionadas em tubos de vidro com diâmetros de $1,5 \mathrm{~cm}$ e $30 \mathrm{~cm}$ de comprimento. Estes tubos foram vedados e conectados aos blocos de envelhecimento previamente aquecidos e estabilizados nas temperaturas anteriormente citadas. Após o período de envelhecimento estabelecido, foram coletadas amostras relativas a cada temperatura, as quais foram acondicionadas em frascos de vidro, selados e armazenados em dessecador, para quantificação posterior da concentração de estabilizante remanescente. ${ }^{13} \mathrm{O}$ período em que as amostras ficaram armazenadas, antes da análise por HPLC, situou-se entre 24 e 48 h, garantindo, assim, nenhuma interferência relevante.

\section{Determinação do tempo de extração do DPA}

Em uma análise preliminar, o tempo de extração a ser adotado no experimento foi determinado. As amostras de propelentes foram deixadas em contato com o solvente por 48 e 96 h. Posteriormente, o extrato foi analisado por HPLC e os percentuais em massa de DPA nas amostras de propelente foram determinados. O procedimento empregado para quantificação foi realizado conforme descrito a seguir, variando-se apenas o tempo de extração conforme relatado anteriormente.

\section{Extração de DPA do propelente}

Para quantificação de DPA no propelente BS REX 1200, fez-se inicialmente a extração do estabilizante. O procedimento de extração consistiu da tomada de $1,0 \mathrm{~g}( \pm 0,1 \mathrm{mg})$ de amostra de BS, em um erlenmeyer com boca e tampa esmerilhada. Em seguida, adicionou-se por meio de uma pipeta volumétrica $50 \mathrm{~mL}$ de diclorometano. $\mathrm{O}$ erlenmeyer tampado foi deixado em repouso por um período de $48 \mathrm{~h}$, sendo agitado a cada $12 \mathrm{~h}$ por meio de um agitador mecânico, durante $30 \mathrm{~min}$. Cabe salientar que o grão propelente em estudo apresentava-se na forma granulada com coloração cinza, sendo que, ao final da extração, manteve sua característica física, não ocorrendo sua dissolução pelo diclorometano.

Ao término do período de extração, uma alíquota de $5 \mathrm{~mL}$ da solução final foi transferida, por meio de uma pipeta volumétrica, para um béquer de $20 \mathrm{~mL}$. O béquer foi levado para um banhomaria e mantido numa temperatura não superior a $40{ }^{\circ} \mathrm{C}$, para a evaporação de todo o diclorometano.

A etapa final consistiu na transferência do resíduo obtido do béquer para um balão volumétrico de $25 \mathrm{~mL}$, utilizando-se uma solução de acetonitrila e água (1:1). Após esta etapa o balão foi avolumado, a solução foi homogeneizada e, a seguir, foi analisada por HPLC. ${ }^{14}$

\section{Quantificação da concentração de DPA (CLAE/HPLC)}

A quantificação de DPA por HPLC foi realizada com um cromatógrafo líquido da marca Waters Associates, empregando-se uma fase móvel composta de acetonitrila e água (1:1), numa vazão de $2 \mathrm{~mL} \mathrm{~min}{ }^{-1}$, utilizando-se uma coluna $\mathrm{C} 18$ de fase reversa da marca Bondapack, detector na região do ultravioleta e comprimento de onda $(\lambda)$ de $280 \mathrm{~nm}$.

Antes da quantificação da concentração de DPA presente no propelente, foi determinado o tempo de retenção do composto nas condições de análise adotadas. Este procedimento consistiu na preparação de um padrão de difenilamina de $10 \mathrm{mg} \mathrm{L}^{-1}$ e análise por HPLC paralelamente a uma solução proveniente da extração de estabilizante da amostra de propelente.

Identificado e determinado o tempo de retenção do DPA, foi realizada a quantificação em quintuplicata da concentração inicial de estabilizante no propelente. Para isto, foi construída uma curva analítica com concentrações entre 5 e $60 \mathrm{mg} \mathrm{L}^{-1}$. Posteriormente, as amostras coletadas durante o envelhecimento isotérmico foram analisadas em triplicata para a determinação da variação da concentração do estabilizante em função da temperatura e do tempo de envelhecimento artificial. ${ }^{14}$

\section{RESULTADOS}

Na Figura 2 é apresentado o gráfico obtido para a identificação e determinação do tempo de retenção do DPA, a partir da sobreposição dos picos resultantes da análise por HPLC da solução padrão e da amostra proveniente da extração do grão propelente BS.

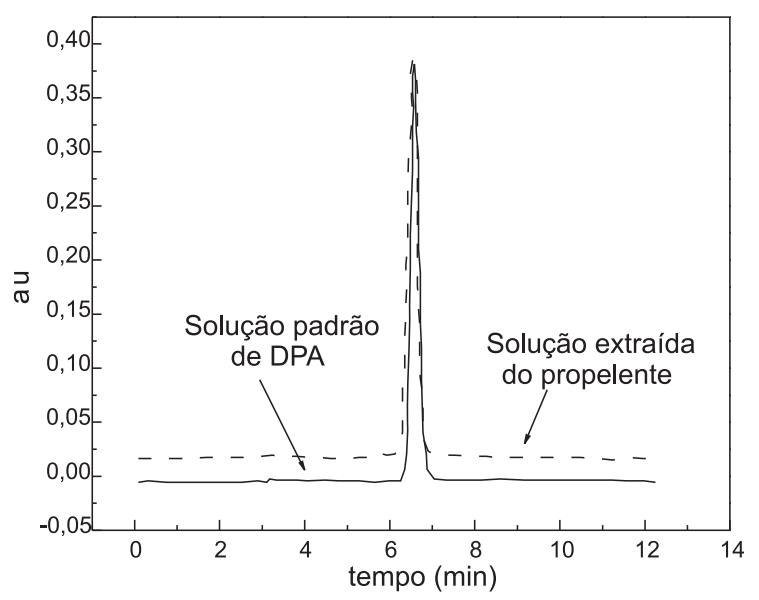

Figura 2. Determinação do tempo de retenção do DPA por HPLC com fase móvel de acetonitrila e água $(1: 1 \mathrm{v} / \mathrm{v})$, em uma coluna $C 18$ de fase reversa e comprimento de onda de ( $\lambda$ ) $280 \mathrm{~nm}$

A Figura 2 demonstra que o tempo de retenção da difenilamina situou-se no intervalo entre 6 a 7 min. Pode-se observar também que no comprimento de onda adotado, relacionado à máxima absorção para o composto, não aparecem picos referentes a produtos de decomposição ou até possíveis impurezas obtidas durante o processo de preparo da amostra. Este fato garantiu uma linha base adequada para a integração da área do cromatograma e, conseqüentemente, a quantificação do DPA.

Em relação à curva analítica obtida para a quantificação de DPA no propelente, a avaliação da linearidade dos pontos obtidos foi realizada utilizando-se o teste da razão entre o sinal (S) e a concentração (Q), definida pela Equação ${ }^{15}$

$(S / Q) \%=\left(\frac{S_{i}-b}{Q}\right) x \frac{100}{a}$

onde $(S / Q) \%$ é o percentual de desvio do ponto em relação à 
inclinação da reta, $S_{i}$ a área do cromatograma relativa à concentração do padrão, $Q$ a concentração do padrão, $b$ o coeficiente linear e $a$ o coeficiente angular.

Determinando-se os valores de $(S / Q) \%$ para cada ponto utilizado na obtenção da curva analítica, tem-se o seu percentual de desvio em relação à reta média. Portanto, adotando-se um intervalo de confiança de $\pm 5 \%$ tem-se que todos os pontos situados entre 95 a $105 \%$ estão dentro de um desvio aceitável em relação à reta média obtida, validando, assim, a curva analítica adotada. Os dados relativos às concentrações dos padrões, as respectivas áreas dos cromatogramas e os valores resultantes de $(S / Q) \%$ em relação à curva analítica utilizada neste trabalho, estão apresentados na Tabela 1.

Tabela 1. Resultados referentes ao estudo da linearidade para os pontos da curva analítica, tendo como base a concentração de DPA e a área média obtida após as injeções no sistema cromatográfico

\begin{tabular}{|c|c|c|}
\hline $\mathrm{Q}\left(\mathrm{mg} \mathrm{L}^{-1}\right)$ & S (área) & $(\mathrm{S} / \mathrm{Q}) \%$ \\
\hline 5,2 & $2,59510^{+5}$ & 103,8 \\
\hline 26,0 & $1,501 \quad 10^{+6}$ & 101,6 \\
\hline 42,0 & $2,32410^{+6}$ & 96,0 \\
\hline 54,8 & $3,23610^{+6}$ & 101,8 \\
\hline 60,0 & $3,49010^{+6}$ & 100,1 \\
\hline
\end{tabular}

Equação da curva analítica DPA: $\mathrm{y}=2,363 \cdot 10^{6} \mathrm{x}-59416,54$; coeficiente de correlação $\mathrm{r}^{2}=0,9990$

A partir da análise do percentual de desvio dos pontos, observa-se que o máximo obtido foi de aproximadamente 4,0\% (42 mg $\left.\mathrm{L}^{-1}\right)$. Além disso, uma análise dos valores de $(S / Q) \%$ em conjunto com o coeficiente de determinação $\left(r^{2}\right)$ 0,9990 deixa claro o excelente ajuste dos dados experimentais a uma equação de reta, cujos parâmetros foram obtidos pelo método dos mínimos quadrados.

$\mathrm{Na}$ Tabela 2 são apresentados os percentuais $(\mathrm{m} / \mathrm{m}) \mathrm{de}$ estabilizante obtidos para os períodos de 48 e 96 h de extração. Conforme pode ser observado em uma análise direta da média aritmética e seus respectivos desvios, os valores obtidos para os percentuais de estabilizante estão relativamente próximos. Para otimizar o tempo total do experimento tendo como base os dados obtidos, foi adotado o período de $48 \mathrm{~h}$ para a extração do estabilizante.

A porcentagem inicial de DPA presente nas amostras do propelente foi determinada antes de submetê-las ao processo de envelhecimento artificial. O processo de extração de DPA e sua determinação foi aplicado às 5 alíquotas da amostra e a média aritmética da porcentagem $(\mathrm{m} / \mathrm{m})$ resultou em 1,02 $\pm 0,01 \%$.

Posteriormente, as amostras de propelente BS coletadas ao longo do processo de envelhecimento foram analisadas e as quantidades de estabilizante remanescentes determinadas.

Tabela 2. Determinação do tempo de extração do estabilizante no propelente

\begin{tabular}{lccc}
\hline $\begin{array}{l}\text { Tempo de extração }(48 \mathrm{~h}) \\
\%(\mathrm{~m} / \mathrm{m}) \text { de estabilizante }\end{array}$ & \multicolumn{2}{l}{$\begin{array}{l}\text { Tempo de extração }(96 \mathrm{~h}) \\
\%(\mathrm{~m} / \mathrm{m})\end{array}$} & de estabilizante \\
\hline 01 & 1,009 & 01 & 1,051 \\
02 & 1,014 & 02 & 1,044 \\
03 & 1,018 & 03 & 1,047 \\
04 & 1,013 & 04 & 1,097 \\
05 & 1,009 & 05 & 1,091 \\
06 & 1,041 & 06 & 1,110 \\
\hline Média & $(1,02 \pm 0,01)$ & Média & $(1,07 \pm 0,03)$ \\
\hline
\end{tabular}

Partindo-se do princípio que o processo de decomposição química siga uma cinética de pseudo-primeira ${ }^{15}$ ordem, que obedece a Equação

$\ln X_{f}=\operatorname{In} X_{i}-k t$

onde $X_{f}$ é a fração remanescente de estabilizante no propelente, $X_{i}$ a fração inicial de estabilizante no propelente, $k=$ Constante cinética e $t$ o tempo.

A partir dos dados coletados, foram gerados os gráficos de ln $X_{f} v s t$ para a determinação da constante cinética relativa a cada temperatura de exposição da amostra. Na Tabela 3 são apresentados para cada amostra, a temperatura de aquecimento, o tempo de envelhecimento e $\ln \mathrm{X}_{\mathrm{f}}$ (fração remanescente de DPA). As Figuras 3 a 5 apresentam a variação de $\ln X_{\mathrm{f}}$ em função do tempo, considerando o modelo de cinética de pseudo-primeira ordem.

Tabela 3. Dados obtidos a partir da quantificação da variação da fração de DPA em função do tempo e da temperatura de envelhecimento

\begin{tabular}{|c|c|c|c|c|c|}
\hline \multicolumn{2}{|c|}{$60^{\circ} \mathrm{C}$} & \multicolumn{2}{|c|}{$70^{\circ} \mathrm{C}$} & \multicolumn{2}{|r|}{$80^{\circ} \mathrm{C}$} \\
\hline $\begin{array}{l}\text { Tempo } \\
\text { (dias) }\end{array}$ & o $\quad \ln X_{f}$ & $\begin{array}{c}\text { Tempo } \\
\text { (dias) }\end{array}$ & $\ln X_{f}$ & $\begin{array}{c}\text { Tempo } \\
\text { (dias) }\end{array}$ & $\ln X_{f}$ \\
\hline 4 & $-4,68 \pm 0,03$ & 7 & $-4,87 \pm 0,05$ & 4 & $-5,04 \pm 0,05$ \\
\hline 7 & $-4,71 \pm 0,06$ & 14 & $-5,02 \pm 0,07$ & 7 & $-5,24 \pm 0,05$ \\
\hline 14 & $-4,79 \pm 0,02$ & 19 & $-5,14 \pm 0,09$ & 14 & $-6,27 \pm 0,10$ \\
\hline 28 & $-4,88 \pm 0,07$ & 28 & $-5,27 \pm 0,07$ & 19 & $-6,81 \pm 0,08$ \\
\hline 36 & $-4,94 \pm 0,06$ & 36 & $-5,40 \pm 0,06$ & 28 & $-7,79 \pm 0,01$ \\
\hline
\end{tabular}

As constantes cinéticas (k) foram calculadas a partir dos respectivos coeficientes angulares conforme a Equação 2 para cada temperatura de trabalho e estão apresentadas na Tabela 4. Conforme se pode observar nesta tabela, o aumento da temperatura leva a um aumento da constante cinética de pseudo-primeira ordem.

Desta forma, a partir da constante cinética relativa a cada temperatura pode-se obter o valor da energia de ativação $\left(E_{a}\right)$ e do fator pré-exponencial utilizando a Equação de Arrhenius na sua forma logarítmica ${ }^{16}$

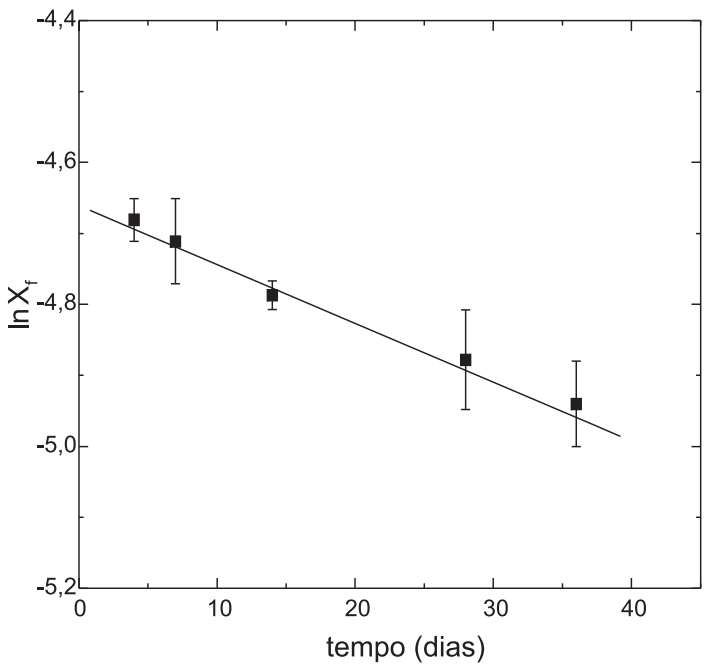

Figura 3. Variação de $\ln X_{f}$ (fração remanescente de DPA) em função do tempo, para o propelente $B S$ envelhecido a $60^{\circ} \mathrm{C}$ 


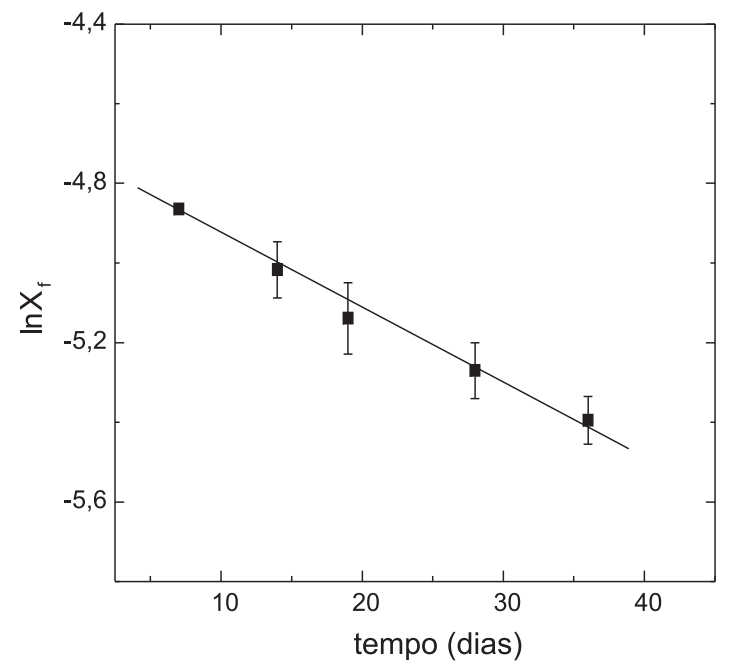

Figura 4. Variação de $\ln X_{f}$ (fração remanescente de DPA) em função do tempo, para o propelente $B S$ envelhecido a $70{ }^{\circ} \mathrm{C}$

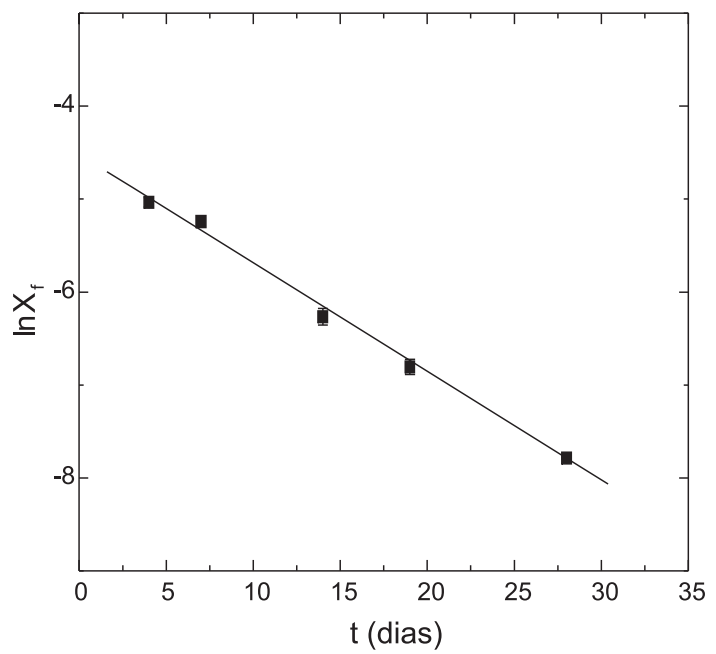

Figura 5. Variação de $\ln X_{f}$ (fração remanescente de DPA) em função do tempo, para o propelente $B S$ envelhecido a $80^{\circ} \mathrm{C}$

Tabela 4. Constantes cinéticas considerando modelo de pseudoprimeira ordem para consumo de DPA no modo isotérmico

\begin{tabular}{lcc}
\hline Temperatura & $\mathrm{R}^{2}$ & $\mathrm{k}\left(\mathrm{dia}^{-1}\right)$ \\
\hline $60{ }^{\circ} \mathrm{C}$ & 0,9852 & $0,008 \pm 0,002$ \\
$70^{\circ} \mathrm{C}$ & 0,9981 & $0,019 \pm 0,002$ \\
$80^{\circ} \mathrm{C}$ & 0,9994 & $0,117 \pm 0,001$ \\
\hline
\end{tabular}

$\ln k=-\frac{E_{a}}{R T} \frac{1}{\ln A}$

sendo " $k$ " a constante cinética para cada temperatura de trabalho, " $E$ " a energia de ativação, " $R$ " a constante universal dos gases $\left(8,3143 \mathrm{~J} \mathrm{~mol}^{-1} \mathrm{~K}^{-1}\right)$; " $\mathrm{T}$ " a temperatura na escala absoluta e "A" o fator pré-exponencial.

A Figura 6 mostra a curva de $1 n \mathrm{k}$ em função de $1 / \mathrm{T}\left(\mathrm{K}^{-1}\right)$.

Os parâmetros cinéticos para consumo de DPA nas amostras do propelente BS REX 1200 foram calculados a partir do gráfico de Arrhenius. A energia de ativação $\left(\mathrm{E}_{\mathrm{a}}\right)$ foi calculada, conforme a Equação 3, a partir do coeficiente angular da reta obtida, ou seja,

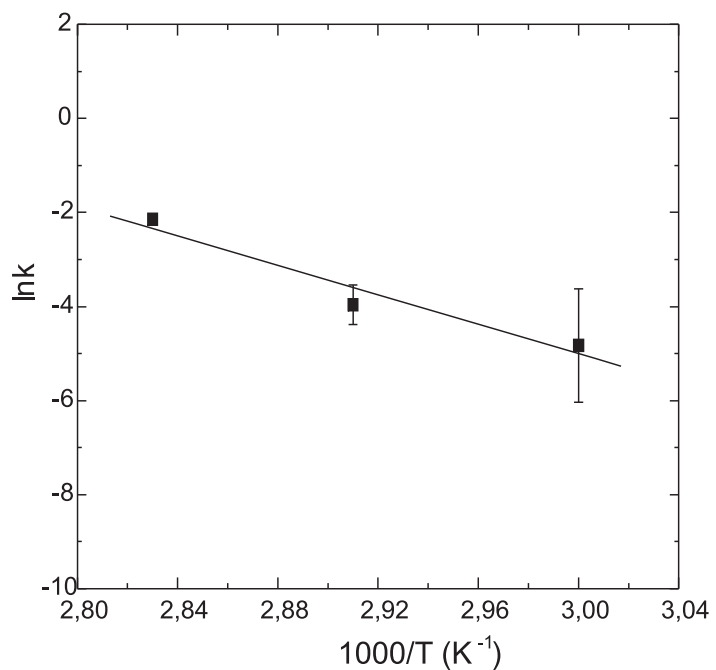

Figura 6. Gráfico de Arrhenius (ln k 1/2s 1/T) para a determinação dos parâmetros cinéticos de consumo de DPA no BS REX 1200, envelhecido a 60, 70 e $80^{\circ} \mathrm{C}$

da razão $E / R$, o fator pré-exponencial (A) foi calculado considerando-se o coeficiente linear (ln A). A $E_{\mathrm{a}}$ encontrada foi de $130 \pm$ $31 \mathrm{~kJ} \mathrm{~mol}^{-1}$ e $\ln \mathrm{A}, 42 \pm 10$.

De modo geral, materiais altamente energéticos, como a nitrocelulose, tendem a ser sensíveis a estímulos térmicos exigindo, assim, maiores cuidados para seu armazenamento. Portanto, procedimentos que permitam obter mais informações quanto à vida útil dos propelentes, para diminuir possíveis riscos de acidentes, são de grande importância.

No Brasil, as normas para avaliação de compostos energéticos são estabelecidas pela Norma Militar Brasileira ${ }^{17}$ apoiada em padrões internacionais adotados pela Organização do Tratado do Atlântico Norte - OTAN. Para propelentes sólidos à base de nitrocelulose a OTAN estabelece como método de avaliação da vida útil a determinação da variação da fração de estabilizante durante armazenamento. Este foi o método adotado no presente trabalho.

O método de extração e posterior quantificação por HPLC adotado neste trabalho demonstrou ser aplicável e adequado ao estudo desenvolvido, em decorrência da obtenção do percentual $(\mathrm{m} / \mathrm{m})$ inicial de DPA no propelente BS de 1,01\%, concordante com o valor confirmado junto ao fabricante do propelente.

Em trabalho semelhante, Jesilavac e Filipovic ${ }^{18}$ obtiveram os parâmetros de Arrhenius para uma amostra de propelente BS utilizando o mesmo princípio, resultando nos valores de $128,3 \pm 2,3 \mathrm{~kJ}$ mol $^{-1}$ para $E_{a}$ e para ln $\mathrm{A}, 29,7 \pm 0,6$. Observa-se que o valor da energia de ativação determinado experimentalmente neste trabalho está próximo ao obtido por Jesilavac e Filipovic ${ }^{18}$ para uma cinética de primeira ordem. A partir dos parâmetros de Arrhenius determinados para o propelente BS REX 1200 calculou-se a constante de velocidade para uma temperatura hipotética de armazenamento de $40^{\circ} \mathrm{C}$ e utilizando-se a equação de tempo de meia-vida para uma cinética de primeira ordem $\left(\mathrm{t}_{1 / 2}=0,693 / \mathrm{k}\right)^{16}$ foi obtido o tempo de 6 anos para que 50\% de DPA presente no BS REX 1200 seja consumido.

Cabe salientar, entretanto, que apesar do método empregado ser uma boa base de informação, dados em relação à vida útil de propelentes não se enquadram apenas em relação à decomposição autocatalítica ocasionada pela depleção do estabilizante. Esta é mais uma ferramenta para auxiliar na avaliação de propelentes à base de nitrocelulose, sendo que análises quanto à sensibilidade, à fricção e ao impacto, ${ }^{19}$ além de mudanças em suas propriedades mecâni- 
cas ocorridas durante armazenamento, são requisitos igualmente importantes para a prevenção de acidentes. ${ }^{20}$

\section{CONCLUSÃO}

Ensaios de envelhecimento no modo isotérmico em temperaturas moderadas, como as empregadas neste trabalho, tendem a ser dispendiosos quanto ao tempo de ensaio. Além disso, entre as variáveis a controlar, observou-se que o monitoramento da temperatura do bloco de envelhecimento ao longo do ensaio, em virtude de possíveis variações na temperatura devido a oscilações de energia da rede elétrica, e a homogeneização da amostra ao longo do ensaio de envelhecimento estão entre os pontos principais.

O método empregado no presente trabalho, apesar de dispendioso, mostrou-se adequado à obtenção de uma base de avaliação do tempo de vida útil de propelente sólido base-simples. Desta projeção resultou um tempo de aproximadamente 6 anos para que 50\% do estabilizante presente no BS REX 1200 seja consumido, em uma temperatura de armazenamento de $40{ }^{\circ} \mathrm{C}$.

\section{AGRADECIMENTOS}

À CAPES pelo apoio financeiro e ao Instituto de Aeronáutica e Espaço (IAE) pelo suporte técnico.

\section{REFERÊNCIAS}

1. Andrade, J.; Iha, K.; Rocco, J. A. F. F.; Bezerra, E. M.; Suárez-Iha, M. E. V.; Pinheiro, G. F. M.; Quim. Nova 2007, 30, 952.

2. Folly, P.; Mäder, P.; Chimia 2004, 58, 374; Paterlini, W. C.; Botelho, E. C.; Rezende, L. C.; Lourenço, V. L.; Rezende, M. C.; Quim. Nova 2002 $25,221$.
3. Auer, N.; Hedger, J. N.; Evans, C. S.; Biodegradation 2005, 16, 229.

4. Lindblom, T.; Christy, A. A.; Libnau, F.; Chemom. Intell. Lab. Syst. 1995, 29, 243; Phillips, R. W.; Orlich, C. A.; Steinberger, R.; J. Phys. Chem. 1955, $59,1034$.

5. Binke, N.; Rong, L.; Zhengquan, Y.; Yuan, W.; Pu, Y.; Rongzu, Hu.; Qingsen, Y.; J. Therm. Anal. Calorim. 1999, 58, 403.

6. Oehrle, S. A.; Propellants, Explos., Pyrotech. 1998, 22, 56.

7. Drzyga, O.; Chemosphere 2003, 53, 809.

8 Lussier, L. S.; Gagnon, H.; Bohn, M. A.; Propellants, Explos., Pyrotech. 2000, 25, 117. .

9. Bohn, M. A.; J. Therm. Anal. Calorim. 2001, 65, 103.

10. Naumov, V. A.; Skii, M. A.; Naumov, A. V.; Samdal, S.; Russ. J. Gen. Chem. J. Gen. Chem. 2005, 75, 923.

11. Bohn, M. A.; Eisenreich, N.; Propellants, Explos., Pyrotech. 1997, 22, 125.

12. Herder, G.; Klerk, W. P. C.; J. Therm. Anal. Calorim. 2006, 85, 169; Evans, G. I.; Gordon, S.; AIAA 1972, 1, 1086; Lindblom, T.; Propellants, Explos., Pyrotech. 2002, 27, 197; Bellamy, A. J.; Bellerby, J. M.; Sammour, M. H.; Propellants, Explos., Pyrotech. 1996, 21, 85.

13. North Atlantic Treaty Organisation; STANAG 4582: qualification of energetic materials, 2004; North Atlantic Treaty Organisation; STANAG 4117: qualification of energetic materials, 1998.

14. Fillipovic, M.; Jelisavac, L. J.; Chromatographia 2002, 55, 239.

15. Prestes, O. D.; Presta, M. A.; Kolberg, I. S.; Zanella, R.; Rossato, S. B.; Penna, N. G.; Hecktheuer, L. H. R.; Quim. Nova, 2007, 30, 18.

16. Atkins, P. Em Princípios de Química - Questionando a Vida Moderna e o Meio Ambiente; Atkins, P.; Jones, L., eds.; Bookamm: Porto Alegre, 2001, cap. 13.

17. Manual Técnico: Material Bélico Armazenamento, Conservação, Transporte e Destruição de Munições, Explosivos e Artifícios T 9-1903/ 1973, [S.1.]: Ministério do Exército.

18. Jelisavac, L.; Filipovic, M.; J. Serb. Chem. Soc. 2002, 67, 103.

19. Silva, G.; Pinheiro, G. F. M.; Iha, K.; Dutra, R. C. L.; Takahashi, M. F. K.; Reis, T. B.; Quim. Nova 2006, 29, 681.

20. Volk, F.; Propellants, Explos., Pyrotech. 1976, 1, 59. 